\title{
On the Precision of a Certain Procedure of Numerical Integration
}

\author{
By Harry D. Huskey \\ With an appendix by Douglas R. Hartree
}

\begin{abstract}
An example of numerical integration is given that shows very systematic effects in the less significant digits. This lack of randomness gives rounding-off errors that exceed the predicted standard deviation by a factor of three.

The example considered in this paper shows that systematic rounding-off errors can occur in numerical integration, irrespective of the number of digits kept in the contributions to the integral. In the appendix this phenomenon is examined, and criteria are set up to detect the cases in which it may arise to a serious extent.
\end{abstract}

\section{Introduction}

The use of numerical methods has led to the study of the accumulation of errors in computations by various people. ${ }^{1}$ In this paper we apply formulas developed by Rademacher to the errors involved in the integration of simultaneous linear differential equations. The system chosen for this application is

$$
\begin{aligned}
& x^{\prime}(t)=y(t), \\
& y^{\prime}(t)=-x(t) .
\end{aligned}
$$

The results of integrating these equations were easily checked by comparison with the sine and cosine tables published by the National Bureau of Standards. ${ }^{2}$

The errors involved in the numerical integration of these equations arise from two sources. One, called the truncation error, arises from replacing the differential equations by difference equations; the other, a round-off error, comes from the rounding-off procedure used in the computation. Formulas developed by Rademacher account for

\footnotetext{
1 F. Schlesinger, Astron. J. 30, 183 (1917); D. Brouwer, Astron. J. 46, 149 (1937); H. Rademacher, On the accumulation of errors in processes of integration on high-speed calculating machines, Proceedings of a Symposium on Large-Scale Digital Calculating Machinery (Harvard University Press, Cambridge, Mass., 1948).

${ }^{2}$ Tables of sines and cosines for radian arguments (National Bureau of Standards, 1940) MT4; Tables of Circular and Hyperbolic sines and cosines for radian arguments (National Bureau of Standards, 1939) MT3.
}

the truncation error. The rounding-off error can be estimated in a statistical manner, provided the dropped digits are randomly distributed. Rademacher suggests that this random property is satisfied provided the increments involved in the integration are not too small. We shall exhibit an integration where this assumption is satisfied, but the dropped digits vary from zero to four and back to zero over a range involving nearly three hundred steps in the integration. This causes the error to increase by a factor of twenty and to become almost three times the standard deviation as given by Rademacher's formulas.

In certain other runs the error exceeds the predicted standard deviation by a small factor. In two of these cases results were tabulated every five or ten steps in the integration and a frequency count of the digits taken. Standard statistical tests indicate that thase numbers did not consist of randomly distributed digits.

These results show that one must be very careful in applying error estimates based on an assumption of randomness. To be safe it is best to use the estimates for the maximum rounding-off error.

\section{Rademacher Theory}

\section{(a) Heun Method}

We now indicate the method of solution studied by Rademacher and give the formulas developed by him. He starts with the system. 


$$
\left.\begin{array}{l}
x^{\prime}(t)=f(x, y) \\
y^{\prime}(t)=g(x, y)
\end{array}\right\}
$$

and the solution is to be found for an interval $t_{0} \leqslant t \leqslant T$ by application of the Heun method. That is, having found $x_{j-1}$ and $y_{j-1}$ as approximations to the solutions at $t_{j-1}=t_{\circ}+(j-1)(\Delta t)$, the following formulas give $x_{j}$ and $y_{j}$.

$$
\left.\begin{array}{c}
x_{j}^{*}=x_{j-1}+\Delta t \cdot f\left(x_{j-1}, y_{j-1}\right) \\
y_{j}^{*}=y_{j-1}+\Delta t \cdot g\left(x_{j-1}, y_{j-1}\right)
\end{array}\right\}
$$

\section{(b) Definitions}

Let us make the following definitions:

(a) Let $x(t), y(t)$ be solutions of eq 1 satisfying the condition that $x\left(t_{o}\right)=x_{o}$ and $y\left(t_{o}\right)=y_{o}$.

(b) Let $x_{j}, y_{j}, j=1,2, \ldots ., n$, be the numbers obtained by successive application of eq 2 and 3 .

(c) Let $\lambda(t), \mu(t)$ be generic notation for solutions of the system

$$
\left.\begin{array}{l}
d \lambda / d t=-(\partial f / \partial x) \lambda-(\partial g / \partial x)_{\mu}, \\
d \mu / d t=-(\partial f / \partial y) \lambda-(\partial g / \partial y)_{\mu}
\end{array}\right\}
$$

(d) Let $u\left(t_{j}\right)=x\left(t_{j}\right)-x_{j}$ and $v\left(t_{j}\right)=y\left(t_{j}\right)-y_{j}$, $j=1, \ldots, n$. The numbers $u\left(t_{j}\right)$ and $v\left(t_{j}\right)$ are a measure of the truncation error in each step of the integration.

\section{(c) Truncation Error}

Rademacher derived the following formulas for the truncation error:

$$
\left.\begin{array}{l}
\lambda(T) u(T)+\mu(T) v\left(T^{\prime}\right) \sim \\
-\frac{1}{12}(\Delta t)^{2}\left[\lambda(t) x^{\prime \prime}(t)+\mu(t) y^{\prime \prime}(t)\right] \mid \begin{array}{l}
T \\
t_{0} \\
\left.-\frac{1}{6}(\Delta t)^{2} \int_{t_{0}}^{T}\left[\lambda^{\prime}(t) x^{\prime \prime}(t)+\mu^{\prime}(t) y^{\prime \prime}(t)\right] d t\right)
\end{array}
\end{array}\right\}
$$

The truncation errors $u(T)$ and $v(T)$ can be separately obtained from eq 5 by applying the proper terminal conditions to the solutions $\lambda(t)$ and $\mu(t)$ of eq 4. For example, $u(T)$ can be found by letting $\lambda(T)=1$ and $\mu(T)=0$.

\section{(d) Rounding-Off Error}

Thus far, it has been assumed that all computations are done exactly. In actual computing, this is not the case. The accumulators or registers of the computing machine accommodate only a limited number of digits. Thus eq 2 and 3 should be written as

$$
\left.\begin{array}{c}
x_{j}^{*}=x_{j-1}+\Delta t \cdot f\left(x_{j-1}, y_{j-1}\right)+\sum_{m} \epsilon_{j m} r_{j m}^{(1)} \\
y_{\mathrm{j}}^{*}=y_{j-1}+\Delta t \cdot g\left(x_{j-1}, y_{j-1}\right)+\sum_{m} \epsilon_{j m} r_{j m}^{(2)}
\end{array}\right\}
$$

The $\boldsymbol{\epsilon}_{j m}$ satisfy $\left|\boldsymbol{\epsilon}_{j m}\right| \leqslant 0.5$. The coefficients $r_{j m}^{(i)}$ depend not only upon the equations to be solved but upon the explicit procedure or order of operations in the process of solution. In the following discussion quantities with bars above them represent the actual numbers stored in the registers or accumulators of the computing machine. Although the analysis can be carried through using eq 6 and 7 , Rademacher makes the simplifying assumption that

$$
\overline{\Delta t f\left(\bar{x}_{j}, \bar{y}_{j}\right)}=\overline{\Delta t \overline{f\left(\bar{x}_{j}, \bar{y}_{j}\right)} .}
$$

This means that he assumes that $f\left(x_{j}, y_{j}\right)$ can be computed sufficiently accurately so that when multiplied by $\Delta t$ any inaccuracies it may have are lost in the digits that are dropped. Thus, eq 7 can be replaced by

$$
\left.\begin{array}{l}
\bar{x}_{j}=\bar{x}_{j-1}+\frac{\Delta t}{2}\left[f\left(\bar{x}_{\mathrm{j}}^{*}, \bar{y}_{\mathrm{j}}^{*}\right)+f\left(\bar{x}_{j-1}, \bar{y}_{j-1}\right)\right]+\boldsymbol{\epsilon}_{j 1} 10^{-k} \\
\bar{y}_{j}=\bar{y}_{j-1}+\frac{\Delta t}{2}\left[g\left(\bar{x}_{\mathrm{j}}^{*}, \bar{y}_{\mathrm{j}}^{*}\right)+g\left(\bar{x}_{j-1}, \bar{y}_{j-1}\right)\right]+\boldsymbol{\epsilon}_{j 2} 10^{-k}
\end{array}\right\}
$$

Note that if the parentheses are removed in eq 8 so that there are four multiplications, then there are four rounding-off terms, say $\epsilon_{\mathrm{j} m}^{s}(s=1,2 ; m=1,2)$.

As in the case of the truncation error let us make the following definition: $\bar{u}_{j}=x_{j}-\bar{x}_{j}$ and $\bar{v}_{j}=y_{j}-\bar{y}_{i}$. 
TABLE 1. Sine-cosine rounding errors

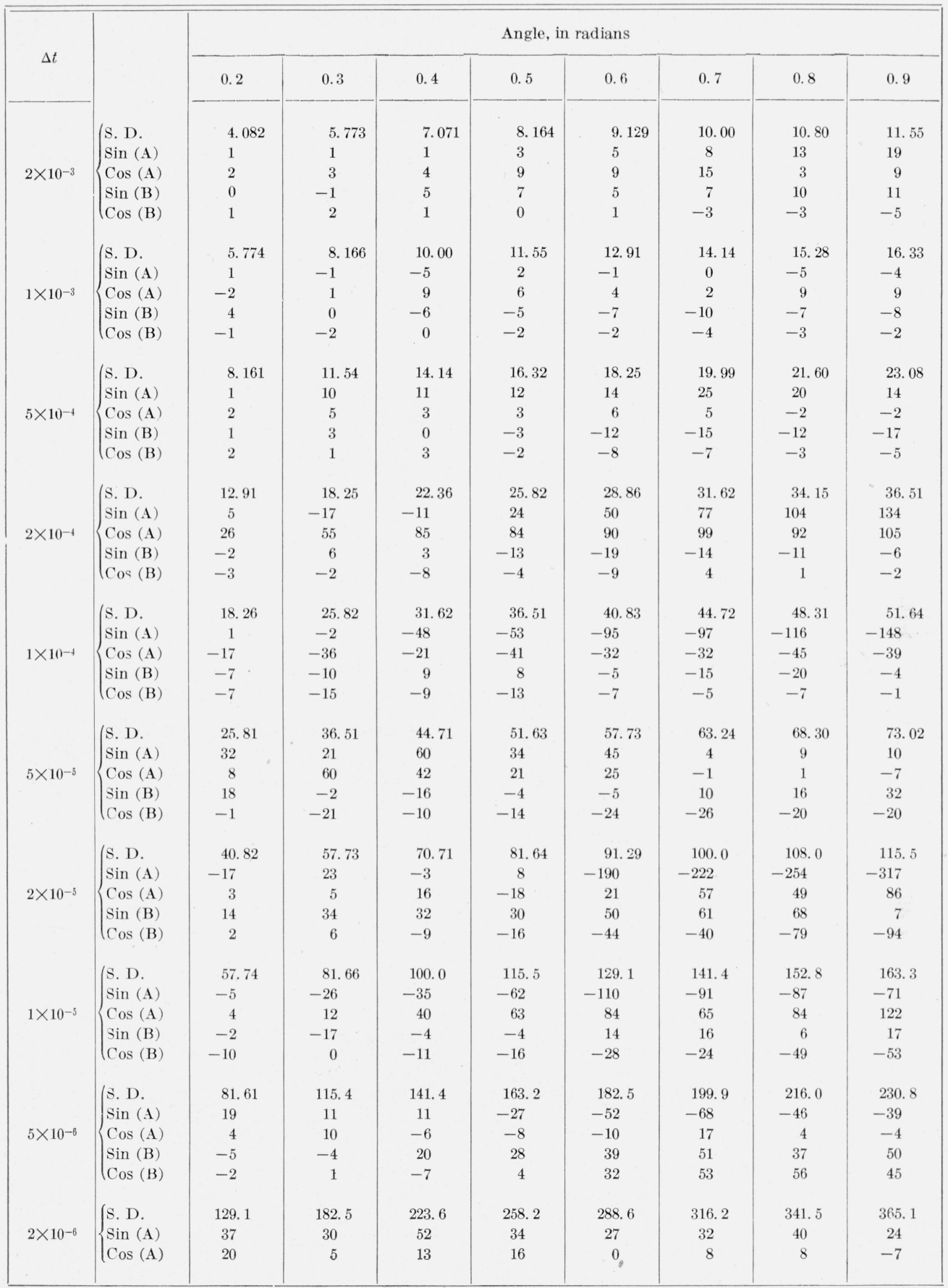


Letting $\bar{u}_{n}=\bar{u}(T)$ and $\bar{v}_{n}=\bar{v}(T)$, the expression for the rounding-off error is

$$
\lambda(T) u(T)+\mu(T) v(T)=-10^{-k} \sum_{j=1}^{n}\left(\epsilon_{j 1} \lambda_{j}+\epsilon_{j 2} \mu_{j}\right) .
$$

From the inequalities $\left|\epsilon_{j m}\right| \leqslant 0.5, m=1,2$, the maximum possible value of the rounding-off error is

$$
\begin{aligned}
\mid \lambda(T) u(T) & +\mu(T) v(T) \mid \leqslant 10^{-k} \sum_{j=1}^{n}\left(\left|\lambda_{j}\right|+\left|\mu_{j}\right|\right) \\
& \sim \frac{10^{-k}}{\Delta t} \int_{t_{0}}^{T}[|\lambda(t)|+|\mu(t)|] d t .
\end{aligned}
$$

However, if the $\boldsymbol{\epsilon}_{j m}, m=1,2$, are random variables then the standard deviation of the rounding-off error is

$$
\begin{gathered}
\sigma[\lambda(T) u(T)+\mu(T) v(T)] \sim \\
\frac{10^{-k}}{\sqrt{3}}(\Delta t)^{-1 / 2}\left[\int_{t_{0}}^{T}\left[\lambda^{2}(t)+\mu^{2}(t)\right] d t\right]^{1 / 2} . \\
\text { II. Example }
\end{gathered}
$$

\section{Sine-cosine Integrations}

To check the theory developed by Rademacher the system

$$
\left.\begin{array}{l}
x^{\prime}(t)=y(t) \\
y^{\prime}(t)=-x(t)
\end{array}\right\}
$$

was integrated on the Electronic Numerical Integrator and Computer. ${ }^{3}$ The range $0.1 \leqslant t \leqslant 0.9$ radians was chosen as the integration interval, since neither function was zero in that interval. (While the function is near zero the increment $\Delta t f(\bar{x}, \bar{y})$ is small and might lead to a systematic effect in the rounding-off.) All computations were done to 10 decimal digits. About 10 values of $\Delta t$ were used ranging from $2 \times 10^{-3}$ to $2 \times 10^{-6}$. A run " $\mathrm{A}$ " was made with the parentheses appearing. in eq 8 removed; this gives four round-offs per integration step. A run "B" was made with the parentheses in; this gives two round-offs per integration step. The results of these runs are tabulated in table 1.

The first entry in each rectangle in table 1 is the run $A$ standard deviation for the respective angle and increment as given by eq 11 . For run $B$ this standard deviation should be divided by two. Underneath are the residual errors (after

\footnotetext{
3 The "ENIAC" was built by the Moore School of Electrical Engineering of the University of Pennsylvania and is now located at the Ballistic Research Laboratcries of Aberdeen Proving Ground.
}

the truncation error is removed) for the various runs and functions. A typical entry (such as the residual error of 15 for the cosine in run $A$, angle equal to 0.7 radians, and increment of $2 \times 10^{-3}$ ) is found as follows:

$$
\begin{aligned}
& \text { Integration result }=0.7648419311 \\
& \begin{array}{r}
\text { Truncation error } \\
\text { as given by eq } 5
\end{array}=\frac{+0.0000002577}{0.7648421888} \\
& \text { True value }=\frac{0.7648421873}{\text { Residual error }}=0.0000000015
\end{aligned}
$$

The most interesting feature in the table occurs

\begin{tabular}{|c|c|c|c|c|}
\hline$t$ & $\begin{array}{c}x \\
\sin t\end{array}$ & $x^{*}$ & $\begin{array}{c}y \\
\cos t\end{array}$ & $y^{*}$ \\
\hline \multirow[t]{2}{*}{0.52250} & $\begin{array}{r}0.4990481273 \\
866574\end{array}$ & $\begin{array}{r}0.4990481273 \\
173314 \\
\end{array}$ & \multirow{2}{*}{$\begin{array}{r}0.8665742703 \\
-499048 \\
-499065\end{array}$} & $\begin{array}{r}0.8665742703 \\
-99810\end{array}$ \\
\hline & 866564 & .4990654587 & & .8665642893 \\
\hline \multirow{2}{*}{.52252} & $\begin{array}{r}4990654586 \\
866564\end{array}$ & $\begin{array}{r}4990654586 \\
173312\end{array}$ & \multirow{2}{*}{$\begin{array}{r}.8665642891 \\
-499065 \\
-499082\end{array}$} & $\begin{array}{r}8665642891 \\
-99814\end{array}$ \\
\hline & 866554 & . 4990827898 & & .8665543077 \\
\hline \multirow[t]{2}{*}{.52254} & $\begin{array}{r}4990827897 \\
866554\end{array}$ & $\begin{array}{r}4990827897 \\
173310\end{array}$ & \multirow{2}{*}{$\begin{array}{r}8665543076 \\
-499082 \\
-499100\end{array}$} & $\begin{array}{r}.8665543076 \\
-99816\end{array}$ \\
\hline & 866544 & 4991001207 & & .8665443260 \\
\hline \multirow[t]{2}{*}{.52256} & $\begin{array}{r}4991001206 \\
866544\end{array}$ & $\begin{array}{r}4991001206 \\
173308\end{array}$ & \multirow{2}{*}{$\begin{array}{r}.8665443258 \\
-499100 \\
-499117\end{array}$} & $\begin{array}{r}.8665443258 \\
-99820\end{array}$ \\
\hline & 866534 & . 4991174514 & & .8665343438 \\
\hline \multirow[t]{2}{*}{.52258} & $\begin{array}{r}4991174513 \\
866534\end{array}$ & $\begin{array}{r}4991174513 \\
173306\end{array}$ & \multirow{2}{*}{$\begin{array}{r}.8665343436 \\
-499117 \\
-499134\end{array}$} & $\begin{array}{r}.8665343436 \\
-99824\end{array}$ \\
\hline & 866524 & . 4991347819 & & .8665243612 \\
\hline .52260 & . 4991347818 & . 4991347818 & .8665243611 & .8665243611 \\
\hline
\end{tabular}
in run $A$ for the sine with an increment of $2 \times 10^{-5}$. For the angle changing from 0.5 to 0.6 radian the residual error jumps from +0.0000000008 to -0.00000000190 . This integration was rerun, and results were printed more frequently. It was found that most of the disturbance occurred between 0.5211 and 0.5264 radian. Table 3 gives the results over this range with printings at every five integration steps, and table 2 exhibits a typical five steps between the values of table 3 .

TABLE 2. Sample step in the sine-cosine integration

$$
\begin{array}{cc}
\Delta t=0.00002 & 0.52250 \leq t \leq 0.52260 \\
x_{i}^{*}=x_{i-1}+\Delta t y_{i-1} & x_{i}=x_{i-1}+\frac{\Delta t}{2}\left[y_{i}^{*}+y_{i-1}\right] \\
y_{i}^{*}=y_{i-1}-\Delta t x_{i-1} & y_{i}=y_{i-1}-\frac{\Delta t}{2}\left[x_{i}^{*}+x_{i-1}\right]
\end{array}
$$


TABLE 3. Sine-cosine integration

$\Delta t=0.00002$

\begin{tabular}{|c|c|c|c|c|c|c|c|}
\hline$t$ & Sine & $\mathrm{E}$ & Cosine & $t$ & Sine & $E$ & Cosine \\
\hline 0.5100 & 0.4881772474 & -4 & 0.8727445090 & 0.5240 & 0.5003474198 & 102 & 0.8658247235 \\
\hline .5110 & .4890497478 & -8 & .8722558955 & .5241 & ! 5004339993 & 107 & .8657746845 \\
\hline .5120 & .4899217591 & -11 & .8717664098 & .5242 & .5005205738 & 112 & .8657246368 \\
\hline .5130 & 4907932802 & -2 & .8712760521 & .5243 & .5006071433 & 117 & .8656745803 \\
\hline .5140 & .4916643102 & -2 & .8707848234 & .5244 & .5006937078 & 122 & .8656245153 \\
\hline .5150 & .4925348499 & -9 & .8702927237 & .5245 & .5007802673 & 127 & .8655744416 \\
\hline .5160 & . 4934048968 & -18 & .8697997538 & .5246 & .5008668218 & 132 & .8655243593 \\
\hline .5170 & 4942744483 & -3 & .8693059143 & .5247 & .5009533713 & 137 & .8654742683 \\
\hline .5180 & .4951435083 & -13 & .8688112054 & .5248 & .5010399158 & 142 & .8654241686 \\
\hline .5190 & . 4960120698 & +2 & .8683156275 & .5249 & .5011264553 & 147 & .8653740603 \\
\hline .5200 & 4968801398 & -18 & .8678191812 & .5250 & .5012129898 & 152 & .8653239433 \\
\hline .5201 & . 4969669193 & -23 & .8677694889 & .5251 & .5012995193 & 157 & .8652738178 \\
\hline .5202 & .4970536938 & -18 & .8677197879 & .5252 & .5013860438 & 152 & .8652236835 \\
\hline .5203 & 4970404633 & -23 & .8676700782 & .5253 & .5014725633 & 157 & .8651735405 \\
\hline .5204 & .4972272278 & -18 & .8676203597 & .5254 & .5015590778 & 162 & .8651233890 \\
\hline .5205 & 4973139873 & -23 & .8675706327 & .5255 & .5016455873 & 167 & .8650732287 \\
\hline .5206 & .4974007418 & -18 & .8675208970 & .5256 & . 5017320918 & 172 & .8650230599 \\
\hline .5207 & 4974874913 & -23 & .8674711525 & .5257 & .5018185913 & 177 & .8649728824 \\
\hline .5208 & .4975742358 & -18 & .8674213995 & .5258 & .5019050858 & 172 & .8649226961 \\
\hline .5209 & .4976609753 & -13 & .8673716378 & .5259 & .5019915753 & 177 & .8648725013 \\
\hline .5210 & 4977477098 & -18 & .8673218673 & .5260 & .5020780598 & 182 & .8648222978 \\
\hline .5211 & .4978344393 & -13 & .8672720881 & .5261 & .5021645393 & 187 & .8647720856 \\
\hline .5212 & 4979211638 & -8 & .8672223004 & .5262 & .5022510138 & 182 & .8647218649 \\
\hline .5213 & .4980078833 & -3 & .8671725039 & .5263 & .5023374833 & 187 & .8646716354 \\
\hline .5214 & 4980945978 & -8 & .8671226987 & .5264 & .5024239478 & 192 & .8646213974 \\
\hline .5215 & .4981813073 & -3 & .8670728850 & .5265 & .5025104073 & 187 & .8645711507 \\
\hline .5216 & 4982680118 & +2 & .8670230625 & .5266 & .5025968618 & 192 & 8645208954 \\
\hline .5217 & .4983547113 & 7 & .8669732313 & .5267 & .5026833113 & 187 & .8644706314 \\
\hline .5218 & .4984414058 & 12 & .8669233916 & .5268 & .5027697558 & 192 & .8644203587 \\
\hline .5219 & .4985280953 & 7 & .8668735431 & .5269 & .5028561953 & 187 & .8643700774 \\
\hline .5220 & . 4986147798 & 12 & .8668236859 & .5270 & .5029426298 & 192 & .8643197874 \\
\hline .5221 & .4987014593 & 17 & .8667738202 & .5271 & .5030290593 & 187 & .8642694889 \\
\hline .5222 & .4987881338 & 22 & .8667239457 & .5272 & .5031154838 & 192 & .8642191816 \\
\hline .5223 & .4988748033 & 27 & .8666740625 & .5273 & .5032019033 & 187 & .8641688658 \\
\hline .5224 & 4989614678 & 32 & .8666241708 & .5274 & .5032883178 & 192 & .8641185413 \\
\hline .5225 & .4990481273 & 37 & .8665742703 & .5275 & .5033747273 & 187 & .8640682081 \\
\hline .5226 & 4991347818 & 42 & .8665243611 & .5276 & .5034611318 & 182 & .8640178664 \\
\hline .5227 & 4992214313 & 47 & .8664744434 & .5277 & .5035475313 & 187 & .8639675159 \\
\hline .5228 & 4993080758 & 52 & .8664245169 & .5278 & .5036339258 & 182 & .8639171569 \\
\hline .5229 & 4993947153 & 57 & .8663745817 & .5279 & .5037203153 & 177 & 8638667892 \\
\hline .5230 & 4994813498 & 62 & .8663246380 & .5280 & .5038066998 & 172 & .8638164129 \\
\hline .5231 & 4995679793 & 57 & .8662746855 & .5281 & .5038930793 & 167 & .8637660279 \\
\hline .5232 & .4996546038 & 62 & .8662247243 & .5282 & .5039794538 & 162 & .8637156344 \\
\hline .5233 & 4997412233 & 67 & .8661747546 & .5283 & .5040658233 & 157 & .8636652321 \\
\hline .5234 & .4998278378 & 72 & 8661247761 & .5284 & .5041521873 & 157 & .8636148213 \\
\hline .5235 & . 4999144473 & 77 & .8660747889 & .5285 & .5042385458 & 162 & .8635644018 \\
\hline .5236 & .5000010518 & 82 & .8660247932 & .5286 & .5043248993 & 167 & .8635139736 \\
\hline .5237 & .5000876513 & 87 & .8659747887 & .5287 & .5044112478 & 172 & 8634635369 \\
\hline .5238 & .5001742458 & 92 & ..8659247757 & .5288 & .5044975913 & 177 & .8634130914 \\
\hline .5239 & .5002608353 & 97 & .8658747540 & .5289 & .5045839298 & 182 & .8633626374 \\
\hline
\end{tabular}

Thus, most of the change in error occurs in an interval of about 0.0053 radian. This represents about 260 integration steps and over a thousand round-offs. In one half of the multiplications, the digit being dropped in the rounding-off process (see the sixth digit in the cosine values of table 3 ) changes gradually from zero up to four and back to zero again.

The errors in the sine values (see column headed
$\mathrm{E}$ in table 3) may be wrong by up to plus or minus five units, since they were obtained by subtracting the integration results (listed in table 3) from nine digit values of the true results taken from Tables of Circular and Hyperbolic Sines and Cosines. ${ }^{4}$

It will be noted that the last digits of the sine values in table 3 , generally speaking, are alternately 3's and 8's. This can be traced to the

4 See footnote 2 
alternate 2's and 7's or 1's and 6's in the fifth digit of the cosine values. This is another warning against unconsidered assumption of randomness in the less significant digits of numbers involved in computations.

Rademacher asserts there will be statistical independence of the dropped digits in the rounding-off process provided $(\Delta t / 2) f\left(\bar{x}_{j-1}, \bar{y}_{j-1}\right)$ and $(\Delta t / 2) f\left(\bar{x}_{j}, \bar{y}_{j}\right)$ differ in the place $10^{-k}$. The example given here shows that Rademacher's condition is not sufficient. In fact, inspection of table 2 shows that the increments $\Delta t / 2 f(x, y)$ may differ in the place $10^{-k}$ and yet be alike for a large number of integration steps in the place $10^{-k-1}$.

There are other examples listed in table 1 leading to large residual errors. For example, consider in run $A$ the sine and cosine values for $\Delta t=2 \times 10^{-4}$ and the sine values for $\Delta t=1 \times 10^{-4}$. More frequent tabulation of results shows a steady increase of residual error with no such jumps as described above.

The author thanks various members of the staff of the Ballistics Research Laboratories and of the Moore School who assisted in running the problem, and extends his appreciation to L. S. Dederick of the Ballistics Research Laboratories, whose permission and cooperation made this paper possible.

\section{Appendix \\ Note on Systematic Rounding-off Errors in Numerical Integration}

\section{By D. R. Hartree ${ }^{5}$}

In this paper, which summarizes the results of a numerical study of truncation and rounding-off errors in the numerical solution of a differential equation by a step-bystep process, Huskey has exhibited a case in which rounding-off errors in a sequence of successive contributions to the solution are systematically of one sign and approximately equal in magnitude, although the leading digit rounded off is the sixth significant figure in each contribution. The result is that the rounding-off errors build up to a total substantially greater than would be estimated in the basis of a random distribution of rounding-off errors in the individual contributions. The purpose of this note is to examine this situation further and to establish criteria for identifying the conditions in which it is likely to occur, so that steps can be taken to deal with it, as for example by carrying an extra significant figure temporarily in the course of the solution.

Consider the numerical evaluation of $\int y d t, k$ decimals being kept in the calculation.

${ }^{5}$ On the staff of the Institute for Numerical Analysis.
Systematic rounding-off errors occur when the leading digit rounded off remains the same in a number of successive contributions to the integral; that is, when for successive contributions, last integer digit of $10^{k+1} y \delta t$ is the same. When this occurs, llast integer digit of $\left.\delta\left(10^{k+1} y \delta t\right)\right]=0$, or [last integer digit of $\left.10^{k+1} \dot{y}(\delta t)^{2}\right]=0$; that is,

$$
10 n-0.5<10^{k+1} \dot{y}(\delta t)^{2}<10 n+0.5,
$$

for some integer $n$. This will usually occur for some value or other of $t$ if

$$
10^{k} \max |\dot{y}|(\delta t)^{2}>1 ;
$$

it will also occur if

$$
10^{k} \max |\dot{y}|(\delta t)^{2}<1 / 10 .
$$

The range $\Delta \dot{y}$ of $\dot{y}$ over which the inequalities (eq. 13) are satisfied is

$$
\Delta \dot{y}=1 /\left[10^{k+1}(\delta t)^{2}\right],
$$

and the number $N$ of intervals required to cover this range is given approximately by

so that

$$
N(\delta t)|\ddot{y}|=\Delta \dot{y}
$$

$$
N=1 /\left[10^{k+1}(\delta t)^{3}|\ddot{y}|\right] .
$$

The accumulation of systematic errors is only serious if $N$ is greater than 3 or 4 , that is, if

$$
4 \cdot 10^{k+1}(\delta t)^{3}|\ddot{y}|<1 ;
$$

for this not to occur

$$
(\delta t)^{3}>1 /\left[4 \cdot 10^{k+1}|\ddot{y}|\right] .
$$

The inequalities (eq. 13 and 17) together provide a criterion for identifying the situations in which accumiulation of systematic rounding-off errors may be dangerous. Such a situation may arise in any numerical integration, not only in the solution of a differential equation, the context in which it was first found by Huskey. The inequality (eq. 17) shows how much more likely it is to arise with small values of the integration interval $(\delta t)$ than with large values.

In the case considered particularly by Huskey, $y=\cos t$, $k=10, \delta t=2 \cdot 10^{-5}, \dot{y}=\sin t$, so that eq 13 becomes

$$
10 n-0.5<40 \sin t<10 n+0.5 ;
$$

this is satisfied for a range of $t$ in the neighborhood of sin $t=1 / 2$, which is just the region in which the phenomenon does occur; and it happens to be particularly marked in this case, since the digit which is rounded off systematically happens to be a 4 over a considerable range. Also

$$
10^{k+1}(\delta t)^{3}|\ddot{y}| \approx 7 \cdot 10^{-4},
$$

so that, from eq. $16, N$ is about 1,400 , and the inequality (eq. 17) is very far from being satisfied; hence it is not surprising that the phenomenon arose in a marked form. The condition (eq. 18) suggests that with the value of $k=10$, the interval length should certainly be greater than $\delta t=10^{-4}$.

Washington,, July 17, 1948. 